\title{
Prototyping a Global Soft X-ray Imaging Instrument for Heliophysics, Planetary Science, and Astrophysics Science
}

\author{
Michael R. Collier ${ }^{1, \star}$, F. Scott Porter ${ }^{1}$, David G. Sibeck ${ }^{1}$, Jenny A. Carter ${ }^{2}$, Meng P. Chiao ${ }^{1}$, Dennis \\ J. Chornay ${ }^{1}$, Thomas Cravens ${ }^{3}$, Massimiliano Galeazzi ${ }^{4}$, John W. Keller ${ }^{1}$, Dimitra Koutroumpa ${ }^{5}$, Kip \\ Kuntz $^{6}$, Andy M. Read ${ }^{2}$, Ina P. Robertson ${ }^{3}$, Steve Sembay ${ }^{2}$, Steven Snowden ${ }^{1}$, and Nick Thomas ${ }^{1}$ \\ ${ }^{1}$ NASA/Goddard Space Flight Center, Greenbelt, Maryland 20771 \\ 2 The University of Leicester, The Department of Physics and Astronomy, Leicester, LE1 7RH, U.K. \\ ${ }^{3}$ University of Kansas, Department of Physics and Astronomy, 1251 Wescoe Hall Dr., Lawrence, Kansas, 66045 \\ ${ }^{4}$ University of Miami, Department of Physics, Coral Gables, Florida 33124 \\ ${ }^{5}$ LATMOS-IPSL/CNRS, 11 Boulevard d'Alembert, 78280 Guyancourt, France \\ 6 The Johns Hopkins University, The Henry A. Rowland Department of Physics and Astronomy, Baltimore, Maryland \\ 21218
}

Received dd mmm yyyy, accepted dd mmm yyyy

Published online later

\begin{abstract}
Key words interplanetary medium; solar-terrestrial relations; solar wind; X-rays, gamma rays; X-rays: diffuse background

We describe current progress in the development of a prototype wide field-of-view soft X-ray imager that employs Lobstereye optics and targets heliophysics, planetary, and astrophysics science. The prototype will provide proof-of-concept for a future flight instrument capable of imaging the entire dayside magnetosheath from outside the magnetosphere. Such an instrument was proposed for the ESA AXIOM mission.
\end{abstract}

(c) yyyy WILEY-VCH Verlag GmbH \& Co. KGaA, Weinheim

\section{Introduction}

Soft X-ray emission from solar wind charge exchange (SWCX) recombination is ubiquitous throughout the solar system. It occurs in planetary atmospheres, comets, interplanetary space, the Earth's exosphere, and likely in supernova remnants and other regions of astrophysical plasmas, making the study of charge exchange truly crossdisciplinary.

Heliophysics: A small fraction of the solar wind mass, momentum, and energy incident upon the terrestrial bow shock enters the Earth's magnetosphere. However, this fraction powers the global magnetospheric disturbances known as geomagnetic storms. Because geomagnetic storms are responsible for some of the most severe space weather disturbances, accurate forecasts from global numerical simulations that incorporate the fundamental physics are essential.

However, such predictive capability requires an instantaneous global view of the overall interaction. Although much has been learned from many years of in-situ spacecraft observations (e.g. Sibeck et al. 1991), these observations are isolated and sporadic. Only simultaneous global observations of the magnetosheath and magnetopause can provide the necessary input for global models (Collier et al. 2009, 2010). Because the solar wind encounters Earth's neutral exosphere in the magnetosheath and cusps, regions

* Corresponding author: e-mail: michael.r.collier@ nasa.gov that map out and provide boundary conditions for Earth's magnetosphere, significant SWCX emission originates from these locations. Both observational evidence (e.g. Carter et al. 2010, 2011) and simulations (e.g. Robertson et al. 2006) show that SWCX soft X-ray imaging will provide this global imaging capability.

Planetary Science: The solar wind-planetary body interaction can be imaged at non-magnetized planets as well as at magnetized planets like Earth. Observations of global SWCX emission at Mars and Venus show that the soft X-ray emissions from both planets are similar in nature to those from Earth proving the feasibility of global terrestrial magnetosheath imaging and indicating that soft $\mathrm{X}$-ray imaging from SWCX emission will be applicable to future planetary missions. The Chandra X-ray observatory in 2001 detected an X-ray halo around Mars due to SWCX (Dennerl et al. 2006; Gunell et al. 2004, 2005; Dennerl 2002; Holmström et al. 2001). Chandra observations in 2006 and 2007 reveal that the Venusian exosphere emits SWCX soft X-rays (Dennerl 2008; Dennerl et al. 2002).

Astrophysics: The soft X-ray emission from charge exchange in the solar system (e.g. Koutroumpa et al. 2009; Robertson et al. 2009; Robertson and Cravens 2003) provides a very significant temporally, spatially, and spectrally varying foreground to all soft X-ray observations from every $\mathrm{X}$-ray observatory. Understanding and modeling this foreground emission in detail is essential for the correct interpretation of data from all Earth-orbiting soft X-ray observa- 


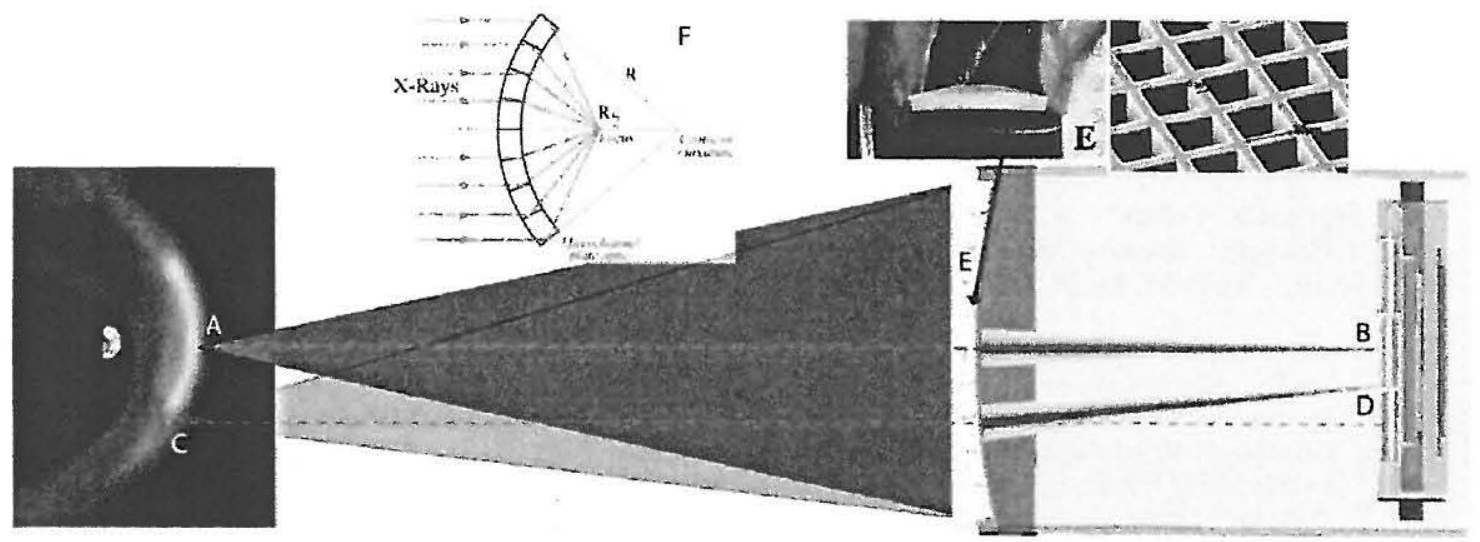

Fig.1 The principle of operation of a wide angle soft X-ray camera: Soft X-ray emission from various locations in Earth's magnetosheath $(A, C)$ are focused onto the detector plane $(B, D)$ by the slumped micropore reflector $(E, F)$.

tories and for maximizing the return from the huge NASA, ESA, and JAXA investments in these missions (Porter et al. 2008). ROSAT, Chandra, XMM-Newton, and Suzaku have all lost significant observing time and errors in scientific interpretation have occurred due to our lack of understanding of this phenomenon (Kuntz 2009).

We report on the design, fabrication, and testing of a laboratory prototype instrument to globally image soft X-ray emission associated with the solar wind's interaction with terrestrial and planetary neutral atoms to map solar wind densities around planets, including the location of the terrestrial magnetopause. Although observations with astrophysics telescopes of SWCX emission near Earth and around Mars and Venus demonstrate the feasibility of global imaging, they are not optimized for observing nearby objects with a wide field-of-view.

\section{Lobster-Eye Optics}

Astrophysicists have developed a variety of instrument techniques for detecting soft X-rays. However, X-ray telescopes using conventional nested mirror optics are optimized for the study of relatively compact objects within a small fieldof-view. The proximity and scale of the magnetopause is such that an X-ray camera designed to image it globally must employ an alternative wide area optic technology such as the micropore (Lobster-Eye) optics design developed by the University of Leicester (Brunton et al. 1997; Fraser et al . 2002) which delivers both wide field-of-view and low mass. The design employs slumped microchannel plates (MCPs) with square channel cross-sections called micropore reflectors (MPRs). The pore sizes on these MPRs are typically 20 or so microns on a side. The square pores provide an array of channels that approximates a Kirkpatrick/Baez system (Kirkpatrick and Baez 1948), but in a small area, increasing the reflecting surface visible to the source and thus the effective area of an optic of a given size. Internal reflections from two orthogonal walls of the square cross-section channel reverse the direction of the two components of the ray's velocity. By slumping the micropore reflector such that the channel axes are perpendicular to the surface of a sphere, the optic will focus $\mathrm{X}$-rays from infinity to an image surface positioned at half the sphere's radius. Figure 1 shows an example of how an instrument using micropore reflectors images the magnetosheath on the focal plane by following two points from the source (A) and (C) onto the focal plane at points (B) and (D) through the reflective micropore $\mathrm{X}$ ray assembly ( $E$ - composed of many tiles shown in the right hand insets) (Price 2001). The focusing properties of the MPR are shown conceptually in inset $(F)$. The divergent beams from points $(A)$ and $(C)$ illuminate the entire optic, but only small angle scattering from the source contributes to the image at points (B) and (D). The reflectivity decreases as a function of angle, shown by shading on the convergent beam to points (B) and (D). The angles are exaggerated for clarity.

Currently micropore reflectors are a possible choice for the optics of the Advanced Telescope for High Energy Astrophysics (Bavdaz et al., 2011). In planetary missions, the micropore optics design will be flown by The University of Leicester as the focusing element of the Mercury Imaging X-ray Spectrometer (MIXS) on the upcoming BepiColombo mission (Fraser et al. 2010; Martindale et al. 2009). The MIXS STM (Structural Thermal Model) has undergone vibration testing, and the optics have successfully passed this vibration testing phase.

\section{Prototype Optics Assembly}

The instrument optics element consists of an array of curved micropore reflectors similar to that shown in (E) of Figure 1. This panel illustrates a slumped micropore reflector and the panel to its right shows a magnified view of the square pores in the reflector whose sides measure 20 microns. 


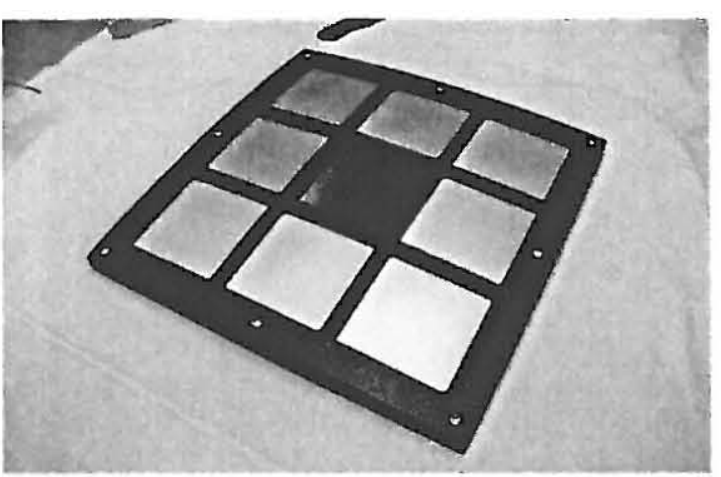

Fig. 2 The $3 \times 3$ optics holder accommodates up to nine $4 \mathrm{~cm} \mathrm{x}$ $4 \mathrm{~cm}$ micropore reflectors with about a $9.2^{\circ}$ field-of-view, side-toside.

We procured from Photonis Corporation (www.photonis.com) $4 \mathrm{~cm} \times 4 \mathrm{~cm}$ slumped micropore reflectors for the prototype. These reflectors have a $75 \mathrm{~cm}$ radius of curvature and a $37.5 \mathrm{~cm}$ focal length. The currently-tested micropore reflectors are uncoated, although we hope to test reflectors coated with platinum soon.

On top of one of the micropore reflectors, Luxel Corporation (luxel.com) bonded a filter constructed of a 2179 Angstrom polyimide layer for UV suppression and a 307 Angstrom aluminum layer for visible suppression. The micropore reflectors serve as a convenient "mesh" for supporting the filter, and this approach proved superior to the standard practice of mounting the filters on a nickel mesh above the detector plane by eliminating the transmission lost to the mesh.

To evaluate how robust the bond between the UV filter and the micropore reflector is to thermal cycling, we performed ten thermal cycles on the filter-bonded MPR using a vacuum oven. The total time the MPR spent above $37.8^{\circ} \mathrm{C}$ was 325 hours 48 minutes. The maximum temperature measured by a thermocouple on the MPR was $185.8^{\circ} \mathrm{C}$. This maximum temperature was held for 21 hours 6 minutes. No visible change in the UV filter or MPR was detected nor was there any evidence of a change in subsequent testing.

A micropore optics holder, shown in Figure 2, was machined to the shape of a portion of a $75 \mathrm{~cm}$ radius sphere. The holder was then populated with the $75 \mathrm{~cm}$ radius of curvature micropore reflector bonded with the filter in the center surrounded by eight aluminum blanks machined to the same shape and size as the micropore reflectors on the periphery. A thin mask holds the micropore reflector and blanks in place. The aluminum optics holder and mask are black anodized for stray light suppression.

\section{Prototype Electronics}

The prototype soft X-ray camera has been designed, built, and tested. It is a self-contained instrument with an anode board, preamplifier and peak-hold board, LVPS board, and

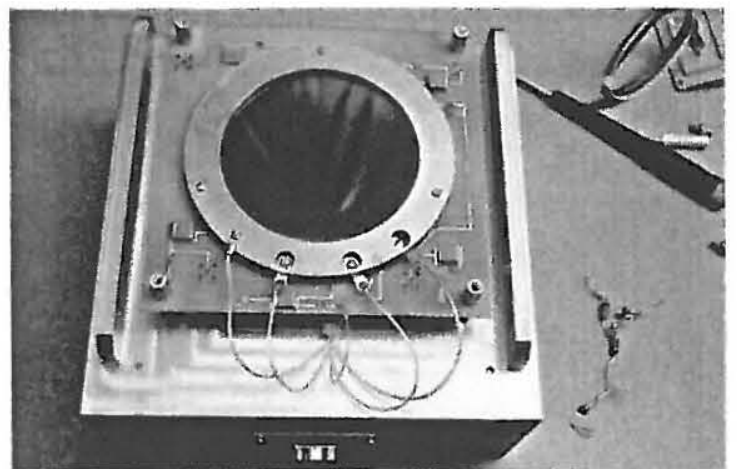

Fig.3 The detector plane is a wedge and strip anode behind a chevron stack of MCPs coated with $\mathrm{KBr}$ for improved soft X-ray response. The anode board is mounted on top of the electronics box.

HVPS board, as well as the optics and housing. With improved venting, some modifications to the peak-hold board, the addition of a C\&DH board, and calibration, the instrument will be flight-capable.

The detector plane, shown in Figure 3 mounted on top of the electronics box, consists of a position sensing wedgeand-strip anode board below a chevron configuration microchannel plate stack coated with $\mathrm{KBr}$ for improved soft $\mathrm{X}$-ray sensitivity.

The wedge and strip anode design measures the position at which an X-ray photon hits the detector plane. To do this it uses the ratio of the amplitudes of two pulses from the wedges which determine the location in one spatial dimension and the ratio of the amplitudes of two pulses from the strips which determine the location in the other spatial dimension. The four raw signals whose amplitudes define the position at which the X-ray photon hit the MCP stack are processed by a peak-hold/preamplifier board that produces square wave output signals proportional to the amplitude of the raw pulses. These square wave signals are subsequently analyzed by a LabView-based GSE (Ground Support Equipment). Some of the LabView functionality will be replaced by the FPGA on the C\&DH board.

An HVPS board using an EMCO Corporation (www.emcohighvoltage.com) C50 supply controlled by a 0 5 Volt analog voltage supplies the high voltage applied to the microchannel plate stack. The specific output voltages required for each stage in the MCP stack are produced by a resistor chain (voltage divider) circuit on the HVPS board.

The camera is powered by an unregulated 28 Volts supplied by a nine pin MDM connector. The 28 Volts is fed directly into the DC-DC converter board. This board which employs two Virginia Power Technology (www.vptinc.com) converters and a filter produces the required voltages, $12 \mathrm{~V}$ and $5 \mathrm{~V}$, to power the HVPS and peak-hold boards as well as the eventual C\&DH board and its FPGA.

All the electronics boards are integrated into a single electronics box shown in Figure 3, with one 9-pin MDM 


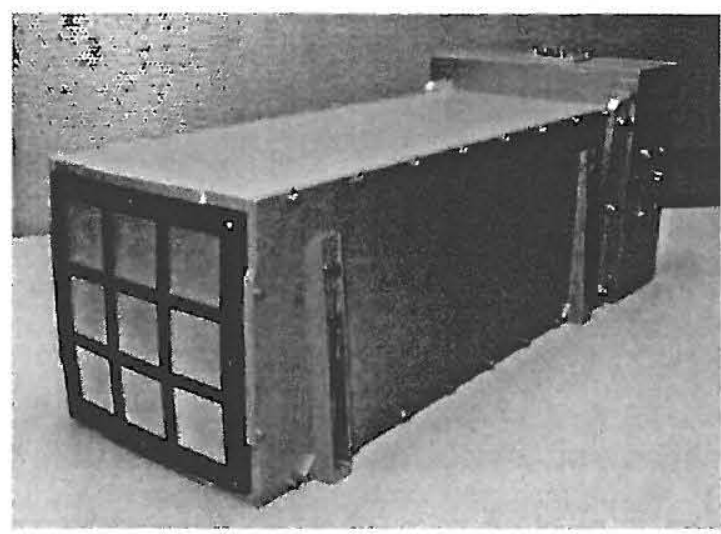

Fig. 4 The integrated instrument prototype with the optics assembly at the front and the electronics box in the back. The electronics box holds the anode, peak-hold, LVPS, and HVPS boards.

Table 1 Prototype Resource Specifications

\begin{tabular}{ccc}
\hline resource & value & notes \\
\hline mass & 7385 grams & no light-weighting \\
power & 3.9 Watts & excludes C\&DH board \\
field-of-view & 9.2 degrees & side-to-side, nine facets \\
envelope & $185 \times 212 \times 520 \mathrm{~cm}$ & $75 \mathrm{~cm}$ roc MPR \\
\hline
\end{tabular}

connector for power and one 15-pin MDM connector for the signals. A third 9-pin MDM connector is used as a high voltage disable plug that shorts out the 12 Volts supplying power to the HVPS board. The current electronics box will also accommodate the additional C\&DH board.

\section{Prototype Integration and Test}

Figure 4 shows the integrated instrument prototype. The housing and electronics box are aluminum plated with gold iridite. Vents will be added to the electronics box and the instrument housing along with a purge fitting for dry nitrogen. Table 1 lists the current prototype resources.

For testing, two 3 foot full nipples (i.e. tubes with flanges at both ends) were mounted to a 2.75 inch conflat flange on a vacuum chamber large enough to accommodate the full instrument shown in Figure 4 to create a six foot beam tube for testing purposes. The vacuum system with the instrument inside achieved vacuum levels in the $10^{-7}$ Torr range. A gate valve with a $\mathrm{Be}$ window on the end of the beam tube allowed instrument testing with an 85 microcurie $\mathrm{Fe}^{55}$ source.

One of the characteristics of the micropore reflector is the generation of a cruciform pattern on the detector plane. Although the six foot beam tube is not long enough to generate rays sufficiently parallel to provide a true test of the optics and the $\mathrm{Fe}^{55}$ test source generates $6 \mathrm{keV}$ (hard rather than soft) X-rays, we still observe a cross-like pattern on the position-sensing anode. Figure 5 shows an example measured point spread function from one screen of the LabView

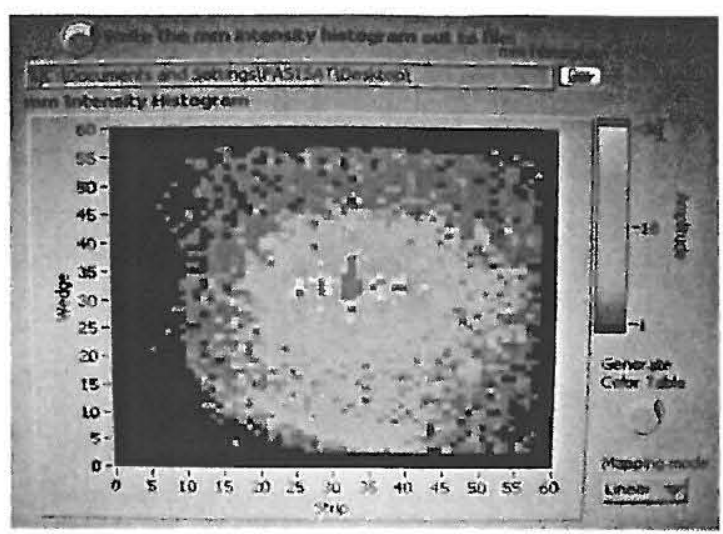

Fig.5 The cruciform point-spread function from the micropore reflector as displayed on the LabView-based GSE.

GSE. The vertical stripes in the horizontal strip direction result from the periodicity of the strips. Future work will include testing the integrated instrument with soft $\mathrm{X}$-rays and with a longer beam tube to produce $\mathrm{X}$-ray beams that are more parallel to provide better characterization of the optics.

\section{Conclusion}

The development of a prototype soft X-ray camera represents an important milestone in establishing a global magnetosheath imaging capability. This instrument demonstrates proof-of-concept in the laboratory and could fly with some planned modifications on an orbital or sub-orbital platform. A soft X-ray camera will play a central role on a future mission, such as the ESA AXIOM mission (BranduardiRaymont et al. 2011).

Acknowledgements. Special thanks to Paul Rozmarynowski for mechanical design support, Kenneth Simms for assembly support, Joseph Kujawski for electrical engineering support, and Norman Dobson for GSE support. The prototype development described in this paper was funded through the Planetary Division and the Astrophysics Division at GSFC through Goddard's Internal Research and Development (IRAD) program.

\section{References}

Bavdaz, M., Rando, N., Wille, E., Wallace, K., Shortt, B., Collon, M., van Baren, C., Pareschi, G., Christensen, F., Krumrey, M., Freyberg, M.: 2011, Proc. SPIE, 8147, 81470C, doi: $10.1117 / 12.893567$

Branduardi-Raymont, G., Sembay, S.F., Eastwood, J.P., Sibeck, D.J., Abbey, T.A., Brown, P., Carter, J.A., Carr, C.M., Forsyth, C., Kataria, D., Kemble, S., Milan, S.E., Owen, CJ., Peacocke, L., Read, A.M., Coates, A.J., Collier, M.R., Cowley, S.W.H., Fazakerley, A.N., Fraser, G.W., Jones, G.H., Lallement, R., Lester, M., Porter, F.S., Yeoman, T.K.: 2011, Exp Astron, doi 10.1007/s10686-011-9239-0 
Brunton, A.N., Fraser, G.W., Lees, J.E., Turcu, I.C.E.: 1997, Appl. Optics, 36, 22, 5461

Carter, J.A., Sembay, S., Read, A.M.: 2010, Mon. Not. Royal Astr. Soc., 402, 867, doi:10.1111/j.1365-2966.2009.15985.x

Carter, J.A., Sembay, S., Read, A.M.: 2011, Astron. Astrophys., 527, A115, doi:10.1051/0004-6361/201015817

Collier, M.R., Abbey, T.F., Bannister, N.P., Carter, J.A., Choi, M., Cravens, T., Evans, M., Fraser, G.W., Hills, H.K., Kuntz, K., Lyons, J., Omidi, N., Porter, F.S., Read, A.M., Robertson, I., Rozmarynowski, P., Sembay, S., Sibeck, D.G., Snowden, S.L., Stubbs, T., Travnicek: 2009, The Local Bubble and Beyond II, edited by K.D. Kuntz, S.L. Snowden, and R.K. Smith, CP1156, 105

Collier, M.R., Sibeck D.G., Cravens, T.E., Robertson, I.P., Omidi, N.: 2010, EOS, Trans., AGU, 91, 213

Dennerl, K.: 2002, Astron. Astrophys., 394, 1119

Dennerl, K.: 2008, Planet. Space Sci., 56, 1414

Dennerl, K., Burwitz, V., Englhauser, J., Lisse, C., Wolk, S.: 2002, Astron. Astrophys., 386, 319

Dennerl, K., Lisse, C.M., Bhardwaj, A., Burwitz, V., Englhauser, J., Gunell, H., Holmström, Jansen, F., Kharchenko, V., Rodriguez-Pascual, P.M.: 2006, Astron. Astrophys., 451, 709

Fraser, G.W. et al.: 2002, Proc. SPIE, 4497, 115

Fraser, G.W. et al.: 2010, Planet. Space Sci., 58, 79

Gunell et al.: 2004, Geophys. Res. Lett., 31, L22801, doi:10.1029/2004GL020953

Gunell, H., Holmström, M., Kallio, E., Janhunen, P., Dennerl, K.: 2005, Adv. Space Res., 36(11), 2057

Holmström, M., Barabash, S., Kallio, E.: 2001, Geophys. Res. Lett., 28, 1287

Kirkpatrick, P., Baez, A.V.: 1948, J. Opt., Soc. Am., 38, 9, 766.

Koutroumpa, D., Lallement, R., Kharchenko, V.: 2009, The Local Bubble and Beyond II, edited by K.D. Kuntz, S.L. Snowden, and R.K. Smith, CP1156, 62

Kuntz, K.D.: 2009, The Local Bubble and Beyond II, edited by K.D. Kuntz, S.L. Snowden, and R.K. Smith, CP1156, 3

Martindale et al.: 2009, Proc. SPIE, 7441, 744115

Porter, F.S., Abbey, T.F., Bannister, N.P., Carter, J.A., Collier, M., Cravens, T., Evans, M., Fraser, G.W., Galeazzi, M., Hills, K., Kuntz, K., Read, A., Robertson, I.P., Sembay, S., Sibeck, D.G., Snowden, S., Stubbs, T., Travnicek, P.: 2008, Space Telescopes and Instrumentation 2008: Ultraviolet to Gamma Ray, Martin J.L. Turner and K.A. Flanagan (eds.), Proc. of SPIE, $70111 \mathrm{~L}$

Price, G.J.: 2001, Microchannel plates in astronomy, Ph. D. Dissertation, Leicester, UK, $180 \mathrm{pp}$.

Robertson, I.P., Cravens, T.E.: 2003, J. Geophys Res., 108, doi:10.1029/2003JA009873

Robertson, I.P., Collier, M.R., Cravens, T.E., Fok, M.-C.: 2006, J. Geophys. Res., 111, doi:10.1029/2006JA011672

Robertson, I.P., Kuntz, K.D., Collier, M.R., Cravens, T.E., Snowden, S.L.: 2009, The Local Bubble and Beyond II, edited by K.D. Kuntz, S.L. Snowden, and R.K. Smith, CP1156, 52

Sibeck, D.G., Lopez, R.E., Roelof, E.C.: 1991, J. Geophys. Res., 96, 5489 